\title{
Highly Efficient Synthesis of Novel Fluorine Bearing Quinoline-4-carboxylic Acid and the Related Compounds as Amylolytic Agents
}

\author{
Mohammed S. T. Makki, Dina A. Bakhotmah", Reda M. Abdel-Rahman \\ Department of Chemistry, Faculty of Science, King Abdulaziz University, Jeddah, Saudi Arabia \\ Email: *dbakhotmah@kau.edu.sa
}

Received January 9, 2012; revised February 17, 2012; accepted February 21, 2012

\begin{abstract}
Highly efficient syntheses of novel fluorine bearing quinoline-4-carboxylic acids and the related compounds had been achieved from cyclocondensation of 2-amino-5-fluorophenyl glyoxylic acid $\mathbf{1}$ with benzoyle asetanilides $\mathbf{2}$ in boiling DMF, to give the target $\mathbf{3}$. Decarboxylation of $\mathbf{3}$ produced 6-fluoro-2-phenyl-3-(substituted amino)-keto-quinolines $\mathbf{4}$, while that reaction underwent refluxing, afforded 7-fluoro-1-(aryl)-3-phenyl-pyrrolo[3,4-c] quinoline-2,9-diones $\mathbf{5}$. Structure of the products has been established from their elemental and spectral analysis. All targets exhibited a high to moderate activity against some Aspergillus fungi as amylolytic agents.
\end{abstract}

Keywords: Amylolytic Activity; Amylolytic Agents; Aspergillus fungi; Efficient Synthesis; Fluoroquinoline; Quinoline-4-carboxylic Acids

\section{Introduction}

Quinoline derivatives are clinically used antimicrobial drugs [1], various heterocyclic systems bearing quinoline moieties distinguish moderate toxicity and CNS (Central Nervous System) stimulants [2,3]. An interesting quinolone derivatives which inhibited HIV-1 replication through interference with the transcription process have been reported, for example; fluoroquinolines (K-12) [8-difluoromethoxy-1-ethyl-6-fluoro-1,4-dihydro-7-[4-(2-methoxyphenyl)-1-pipernzinyl] -4-oxo-quinoline-3-carboxylic acid] and K-37 [7-(3,4-dihydro-4-pheny-l-piperidnyl)-1,4dihydro-6-fluoro-1-methyl-8-trifluoromethyl-4-oxo-quinoline-3-carboxylic acid] [4-8], (Figure 1).

Quinoline nucleus is significant in designing a number of pharmacologically active compounds [9-16]. In continues to our investigation towards quinolines synthesis [17-19] we successfully synthesized a number of fluorine bearing quinoline-4-carboxylic acids in high yield. These compounds are efficiently investigated on various fungi as amylolytic agents.

\section{Results and Discussion}

The cyclocondensation of 2-amino-5-fluorophenyl glyoxylic acid $\mathbf{1}$ [obtained from basic treatments of 5-fluoroisatin] with benzoyl asetanilide derivatives 2 [obtained from preheated ethyl benzoyl acetate with halogenated

*Corresponding author. aromatic amines and or sulfa drugs] in DMF [20-25], yielded the target compounds 6-fluoro-2-phenyl-3-(arylamino)-oxo-quinoline-4-carboxylic acids 3a-d (Scheme 1).

The decarboxylations of compounds 3a-d by gentle warming with aqueous $\mathrm{K}_{2} \mathrm{CO}_{3}$ at $40^{\circ} \mathrm{C}-60^{\circ} \mathrm{C}$ for $10 \mathrm{~min}$ produced the quinoline derivatives $\mathbf{4 a - d}$, while refluxing the potassium salt of compound 3 with $\mathrm{K}_{2} \mathrm{CO}_{3}$ solution for $2 \mathrm{~h}$ gave 7-fluoro-1-(arylamino)-3-phenylpyrrolo[3, 4-c]quinoline-2,9-dione (5a-d). Thus, Compounds 3 were undergoing intramolecular heterocyclization via ring closure reaction by loss of one mole of $\mathrm{KOH}$ to give a more stabilized fused Reterobicyclic systems 5 (Scheme 2).

Structures of novel compounds have been deduced from their elemental analysis and spectral measurements. The IR spectra of compounds $\mathbf{3}$ showed broad bands at $3300-2800 \mathrm{~cm}^{-1}$ that attributed $\mathrm{OH}$ and $\mathrm{NH}$ functional groups in addition to sharp bands at 1700, 1630 and 1220 $\mathrm{cm}^{-1}$ due to presence of carboxylic acid, amide and C-F groups. ${ }^{1} \mathrm{HNMR}$ spectra showed the $\mathrm{COOH}$ and NHCO protons at $\delta 14$ and $\delta 11.5$ respectively, while characteristic protons of aryl and phenyl appeared at $\delta 8.5-7.1 \mathrm{ppm}$. ${ }^{13} \mathrm{CNMR}$ spectra showed carbons of $\mathrm{COOH}, \mathrm{CONH}$, $\mathrm{C}=\mathrm{N}, \mathrm{C}-\mathrm{F}$ and aryl at 168, 162, 151, 140 and 130-125 ppm respectively. Thus, compounds 4 showed the absence of $\mathrm{COOH}$ functional groups due to lack of $\mathrm{OH}$ and carbonyl peaks.

The structure of compound $\mathbf{5}$ was established from that 


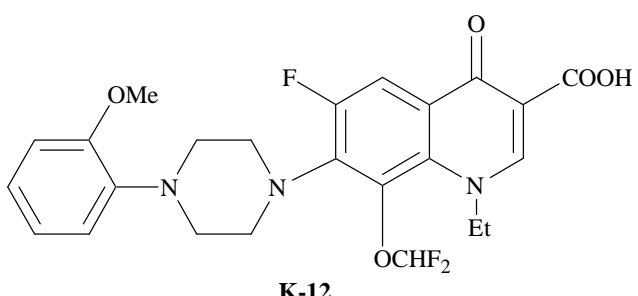

K-12

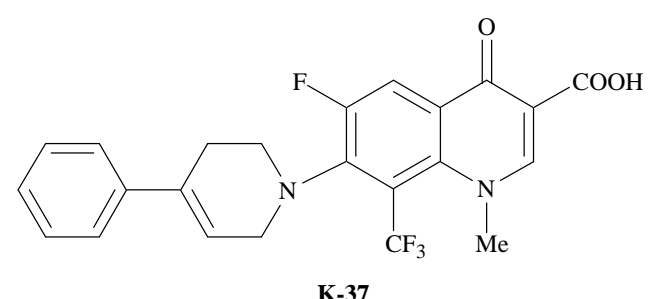

K-37

Figure 1. The chemical structure of fluoroquinolines K-12 and K-37.
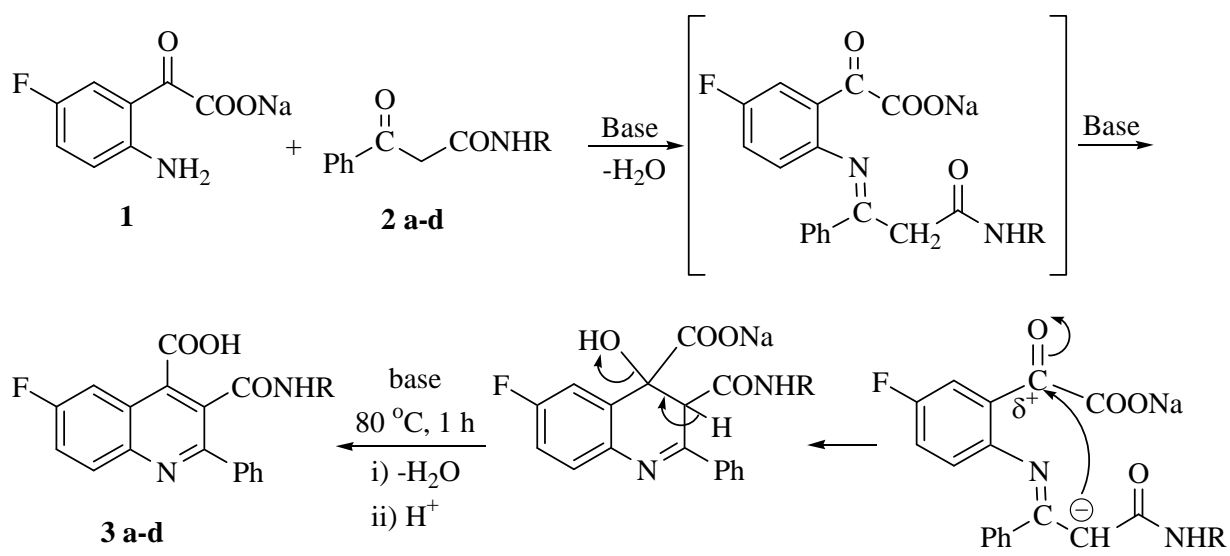

$\mathrm{R}=\mathrm{a}$<smiles>Fc1ccc(I)cc1</smiles>

c)<smiles>Cc1ccc(S(=O)(=O)Nc2nccs2)cc1</smiles>

b)<smiles>Cc1ccc(Cl)c(Cl)c1</smiles>

d)<smiles>Cc1ccc(S(=O)(=O)Nc2nccc(C)n2)cc1</smiles>

Scheme 1. The synthesis of key compounds 3a-d.<smiles>[R]NC(=O)c1c(-c2ccccc2)nc2ccc(F)cc2c1C(=O)O</smiles>

\section{$\underset{40-60{ }^{\circ} \mathrm{C}, 10 \mathrm{~min}}{\stackrel{5 \% \text { aq }}{\mathrm{K}} \mathrm{CO}_{3}}$} $-\mathrm{CO}_{2}$<smiles>NC(=O)c1cc2cc(F)ccc2nc1-c1ccccc1</smiles>
4 a-d

$5 \%$ aq $\mathrm{K}_{2} \mathrm{CO}_{3}$,

1) $\mathrm{rt}, 1 \mathrm{~h}$

2) $80-100{ }^{\circ} \mathrm{C}, 2 \mathrm{~h}$<smiles>[R]N1CC([O-])(O)c2c(c(-c3ccccc3)nc3ccc(F)cc23)C1=O</smiles><smiles>[R][NH+]1C(=O)c2c(-c3ccccc3)nc3ccc(F)cc3c2C1([O-])O</smiles>
$\stackrel{-\mathrm{KOH}}{\longrightarrow}$<smiles>[R]N1C(=O)c2c(-c3ccccc3)nc3ccc(F)cc3c2C1=O</smiles>

Scheme 2. Synthetic strategy towards compounds 5a-d.

IR spectrum via lack of $\mathrm{OH}$ and $\mathrm{NH}$ peaks. While the carbonyl groups of pyrroloquinoline gave two sharp peaks at $\delta 1720$ and $1680 \mathrm{~cm}^{-1}$ in addition to different 1HNMR and ${ }^{13}$ CNMR spectrum.
Study of IR spectra of the synthesized compounds in both solid and solution state $\left(\mathrm{CHCl}_{3}\right)$ showed 1) Lack of the absorption bands, of $\mathrm{OH}$ and $\mathrm{NH}$, while the $\mathrm{C}=\mathrm{O}$ and $\mathrm{C}-\mathrm{F}$ bands moved to a higher wave length in a solution of 
compounds 3 due to the interaction between the molecule and the solvent. 2) Compounds 5, 4 solutions showed limited effect on IR wave length. The presence of carboxylic acid, amide and fluorine groups in compounds $\mathbf{3}$ should have an important activity towards tested organisms via hydrogen bonding or electrostatic interaction with the acceptor centre on the surface of microorganism.

The amylolytic activity of the synthesized fluoroquinolines was studied on Six types of Aspergillus fungi; Aspergillus flavus, Aspergillus fumigain, Aspergillus nidulans, Aspergillus niger, Aspergillus ferreus and Aspergillus terricola. The experimental: Culture medium used for amylase production was prepared according to the described method [26]. Amylase activity of compounds 3a-d, 4a-d and 5a-d was assayed at the $\mathrm{pH} 4.6$ and at $37^{\circ} \mathrm{C}[27,28]$. The final $\mathrm{pH}$ and the mycelia dry weight for each fungus was also recorded, (Table 1). Under the investigation conditions employed, mycelia growth depends on the nature of organism of the tested Aspergillus fungi that showed moderate to lethal mycelia growth in the acidic range. Most organisms have been reported to produce acidic amylases [29,30].

The effect of the compounds prepared on the fungi amylolytic enzyme was studied by incubating these substances with the enzyme solution at $37^{\circ} \mathrm{C}$ for $30 \mathrm{~min}$. Followed by addition of the amylase production (substrate) and estimating the activity in mg reducing sugar/wt of reaction mixture. It was taken into consideration that such substance may affect the enzyme or the substrate or both and enhance the reaction. On the other hand, the enzyme activity of Aspergillus fungi; was inhibited by action of compounds, 3a-d, 5a and 5c, mostly due to fluorine and sulfathinzole moieties.

The pyrroloquinolines 5 showed more effect towards the tested fungi in comparison with $\mathbf{4}$, which may be attributed to electrons asymmetrically distributed on different sites of compounds 5. In addition, replacement of the carboxylic acid group at position 4 in compound 3 by hydrogen atom as in compound $\mathbf{4}$ led to lower effects of compounds 4 over 3 .

Finally, the high effect of the compound $3 \mathbf{a}$ in comparison with 3b-d, 4 and 5, mainly attributed to full fluorine atoms and carboxylic group. Thus, the compound $\mathbf{3 a}$ can be a good chemical probe on amylolytic activity on some Aspergillus fungi.

\section{Conclusions}

Fluoroquinolines of general structures $\mathbf{3 , 4}$ and 5 were efficiently synthesized. Most of these compounds showed significant activity against some Aspergillus fungi as amylolytic agents. The higher activity over other tested compounds was registered for compounds $4-\mathrm{F}-\mathrm{C}_{6} \mathrm{H}_{4}$ (3a, 4a, 5a) and sulfa thiazole moieties (3-c, 4-c, 5-c). These novel molecules possibly will be a new promising anti fungal hit compounds. While, the presence of $3,4-\mathrm{Cl}_{2-}$ $\mathrm{C}_{6} \mathrm{H}_{3}$ and absence of carboxylic group was an inhibited activity. This observation may indicate that biological target is different from that of halogen type. Furthermore, compound discussed herein showed no structural similarity to any other compounds active against some Aspergillus fungi, this may indicate that a novel fluorinated quinoline acts by a new mechanism of action.

In progress, a structural modification of compounds $\mathbf{3}$, 4 and 5 in orders to enhance its efficacy against some Aspergillus fungi. This novel and simple method enjoys higher efficiency, environmental-friendly characteristics in addition to the importance of the organic C-F group in medicinal chemistry; due to it is metabolic transformations in vivo [31,32].

\section{Experimental Section}

General procedures. The melting points were determined with a Gallenkamp melting point apparatus and were uncorrected. NMR spectra were obtained on a Bruker Avance (400 MHz) spectrometer. Chemical shifts were expressed in $\delta$ (ppm) units using DMSO-d $\mathrm{d}_{6}$ as a solvent. Elemental Analysis was performed with a LECO CHNSFCl-932 instrument. IR spectra were recorded with a FT-IR Bomem MB 104 using nujol mulls and $\mathrm{NaCl}$ cells. Compounds $\mathbf{1}$ and $\mathbf{2}$ were prepared according to previously described procedures [22,23].

Synthesis of fluorinated quinoline- 4 carboxylic acids (3a-d). An equimolar mixture of 2-amino-5-fluoropheny-lglyoxylic acid $\mathbf{1}$ and benzoyl asetanilide derivative 2a-d in DMF was refluxed for $4 \mathrm{~h}$ then poured over ice. The resulting solid was filter, washed with water and crystalliz from THF. The resulting solid was identified as fluorinated quinoline-4-carboxylic acids 3.

6-Fluoro-2-phenyl-3-[(4'-fluorophenyl)amino]-oxoquinoline-4-carboxylic acid (3a). Yellow crystals, Yield $70 \%$; m.p. $138^{\circ} \mathrm{C}-140^{\circ} \mathrm{C}$ decomposition; (Found: C, 68.15; H, 3.42; N, 6.84; F, 9.49. $\mathrm{C}_{23} \mathrm{H}_{14} \mathrm{~N}_{2} \mathrm{~F}_{2} \mathrm{O}_{3}$ requires C, 68.14; H, 3.45; N, 6.91; F, 9.62\%); IR (Nujol mull), v $\left(\mathrm{cm}^{-1}\right): 3300$ - 2800 (br, OH, NH), $1710(\mathrm{C}=\mathrm{O}) 1620$ (CONH), 1580 (C=N) 1230 (C-F), 700, (C-Cl). ${ }^{1} \mathrm{H}$ NMR $\left(\mathrm{DMSO}_{6} \mathrm{~d}_{6}\right), \delta: 14.0(\mathrm{~s}, 1 \mathrm{H}, \mathrm{COO} \underline{\mathrm{H}}), 11.21$ (s, $1 \mathrm{H}$, CONH), 8.52, 8.20 (each d, $1 \mathrm{H}, 1 \mathrm{H}, J=8.2$ and $7.5 \mathrm{~Hz}$ ), 8.12(m, 1H), 7.93 - 7.90 (each $\mathrm{m}, 2 \mathrm{H}$, aryl), and 7.80, 7.40 (each $\mathrm{m}, 2 \mathrm{H}$ and $5 \mathrm{H}$, aryl and phenyl protons). ${ }^{13} \mathrm{C}$ NMR (DMSO- $\left.\mathrm{d}_{6}\right), \delta$ : 167.5 and $162.5(2 \mathrm{C}=\mathrm{O}), 151.76$ and 151.0 (C-F), 140.6 (C-N), 136.2, 134.9, 130.2, 128.5, 128.2, 127.6, 126.8, 124.2 and 122.1 (aryl and phenyl carbons).

6-Fluoro-2-phenyl-3-[(2',4'-dichlorophenyl)amino]o xoquinoline-4-carboxylic acid (3b).

Yellow crystals, Yield $75 \%$; m.p. $118^{\circ} \mathrm{C}-120^{\circ} \mathrm{C}$ de- 
Table 1. Amylolytic activity.

\begin{tabular}{|c|c|c|c|c|c|c|c|c|c|c|c|c|c|c|c|}
\hline \multirow[b]{2}{*}{$\begin{array}{c}\text { Tested } \\
\text { Organisms }\end{array}$} & \multirow[b]{2}{*}{$\begin{array}{l}\text { Final } \\
\mathrm{pH}\end{array}$} & \multicolumn{14}{|c|}{ Amylolytic Activity (mg reducing sugar/reaction mixture) } \\
\hline & & $\begin{array}{c}\text { Mycelial dry } \\
\text { wt, (mg/50 } \\
\text { ml culture } \\
\text { med.) }\end{array}$ & Control & 3a & $3 \mathbf{b}$ & 3c & 3d & $4 a$ & $4 \mathbf{b}$ & 4c & $4 d$ & $5 a$ & $5 \mathbf{b}$ & $5 c$ & $5 d$ \\
\hline $\begin{array}{l}\text { Aspergillus } \\
\text { flavus }\end{array}$ & 3.8 & 115 & 3.89 & 2.11 & 3.15 & 2.50 & 3.55 & 3.5 & 3.80 & 3.60 & 3.78 & 3.40 & 3.55 & 3.18 & 3.70 \\
\hline $\begin{array}{l}\text { Aspergillus } \\
\text { fumigain }\end{array}$ & 3.5 & 740 & 6.48 & 5.21 & 6.11 & 4.95 & 6.31 & 5.01 & 5.40 & 5.90 & 6.10 & 5.11 & 5.30 & 5.10 & 5.00 \\
\hline $\begin{array}{l}\text { Aspergillus } \\
\text { nidulans }\end{array}$ & 4.0 & 720 & 5.18 & 4.55 & 4.90 & 4.81 & 5.00 & 5.00 & 5.15 & 5.10 & 5.18 & 4.90 & 4.99 & 4.80 & 5.00 \\
\hline $\begin{array}{l}\text { Aspergillus } \\
\text { niger }\end{array}$ & 3.4 & 153 & 3.37 & 3.01 & 3.80 & 2.99 & 3.99 & 3.11 & 3.25 & 3.12 & 3.30 & 3.11 & 3.20 & 3.15 & 3.28 \\
\hline $\begin{array}{c}\text { Aspergillus } \\
\text { terreus }\end{array}$ & 3.6 & 135 & 5.18 & 4.80 & 4.95 & 4.70 & 5.10 & 4.70 & 5.00 & 4.80 & 5.10 & 4.88 & 5.18 & 4.91 & 5.18 \\
\hline $\begin{array}{c}\text { Aspergillus } \\
\text { terricola }\end{array}$ & 3.9 & 805 & 5.70 & 5.11 & 5.35 & 5.01 & 5.50 & 5.40 & 5.70 & 5.40 & 5.70 & 5.30 & 5.56 & 5.20 & 5.11 \\
\hline
\end{tabular}

composition; (Found: C, 59.94; H, 2.81; N, 6.12; F, 4.11; $\mathrm{Cl}$, 15.41. $\mathrm{C}_{23} \mathrm{H}_{13} \mathrm{~N}_{2} \mathrm{FCl}_{2} \mathrm{O}_{3}$ requires $\mathrm{C}, 55.90 ; \mathrm{H}, 2.85 ; \mathrm{N}$, 6.15; F, 4.17; Cl, 15.60\%); IR (Nujol mull), $v\left(\mathrm{~cm}^{-1}\right)$ : 3300 - 2800 (br, $\mathrm{OH}$ and $\mathrm{NH}), 1710(\mathrm{C}=\mathrm{O}), 1620$ (CONH), 1580 (C=N), 1230 (C-F), 700 (C-Cl). ${ }^{1} \mathrm{H}$ NMR (DMSO-d $\mathrm{d}_{6}$ ), $\delta: 14.12(\mathrm{~s}, 1 \mathrm{H}, \mathrm{COO} \underline{\mathrm{H}}), 11.50(\mathrm{~s}, 1 \mathrm{H}$, CON $\underline{\mathrm{H}}$ ), 8.41, 8.01 (each d, $1 \mathrm{H}, 1 \mathrm{H}, J=8.3$ and $7.8 \mathrm{~Hz}$ ), 8.12 (m, 1H) 7.95 - 7.92 (each m, 2H, Aryl), 7.70, 7.30 (each $\mathrm{m}, 1 \mathrm{H}$ and $5 \mathrm{H}$, aryl and phenyl protons). ${ }^{13} \mathrm{C}$ NMR (DMSO- $\mathrm{d}_{6}$ ), $\delta$ : $168.0(\mathrm{COOH}), 163.1(\mathrm{CONH}), 151.0$ (C-F) 148.5 (C-Cl), 140.8 (C-N), 136, 135, 130, 128.5, 126.9, 125.8, 124.1 and 123.9 ppm (Aryl and Phenyl carbons).

6-Fluoro-2-phenyl-3-[(4'-heteroaryl sulfonamidephenyl) amino] oxoquinoline-4-carboxylic acid (3c). Orange crystals, Yield $78 \%$; m.p. $170^{\circ} \mathrm{C}-172^{\circ} \mathrm{C}$ decomposition; (Found: C, 56.30; H, 3.05; N, 10.07; F, 3.42; S, 11.55. $\mathrm{C}_{26} \mathrm{H}_{17} \mathrm{~N}_{4} \mathrm{FS}_{2} \mathrm{O}_{5}$ requires: C, 56.93, $\mathrm{H}, 3.10$; N, 10.21; F, 3.46, S, 11.67). IR (Nujol mull), $v\left(\mathrm{~cm}^{-1}\right): 3270-$ 2750 (br, OH and $\mathrm{NH}), 1700(\mathrm{C}=\mathrm{O}), 1610(\mathrm{CONH})$, $1570(\mathrm{C}=\mathrm{N}), 1540(\mathrm{C}=\mathrm{N}), 1350\left(\mathrm{SO}_{2} \mathrm{NH}\right), 1230(\mathrm{C}-\mathrm{F})$ $\mathrm{cm}^{-1} .{ }^{1} \mathrm{H}$ NMR (DMSO-d $\left.\mathrm{d}_{6}\right), \delta: 14.50(\mathrm{~s}, \mathrm{COOH}), 13.10(\mathrm{~s}$, $\mathrm{CONH}), 11.50\left(\mathrm{~s}, \mathrm{SO}_{2} \mathrm{NH}\right), 8.81,8.72(\mathrm{~d}, 2 \mathrm{H}$, thiazole), 8.40, 8.31 (d, $1 \mathrm{H}, J=8.3 \mathrm{~Hz}), 8.22-8.10(\mathrm{~m}, 2 \mathrm{H}, o-$ fluorophenyl), 7.93 - 7.70, 7.60 - 7.41 (each $\mathrm{m}, 4 \mathrm{H}, 1.4-$ distributed phenyl). ${ }^{13} \mathrm{C}$ NMR (DMSO- $\mathrm{d}_{6}$ ), $\delta: 170.0$, 167.6 (COOH and CONH), 155.0 (N-C-S) 151.1, 150.2 (C-F, C=N), 148.7, 138.2, 136.3, 134.9, 130.1, 128.9, 128.17, 127.91, 126.84, 125.7, 124.31, 122.11 (hetero, aryl and phenyl carbons).

6-Fluoro-2-phenyl-3-[(4'-heteroaryl sulfonamidophenyl)amino]oxo-quinoline-4-carboxylic acid (3d). Yellow crystals, Yield $75 \%$; m.p. $103^{\circ} \mathrm{C}-105^{\circ} \mathrm{C}$ decomposition; (Found: C, 59.96; H, 3.55; N, 12.49; F, 3.36; S, 5.60. $\mathrm{C}_{28} \mathrm{H}_{20} \mathrm{~N}_{5} \mathrm{FSO}_{5}$ requires $\mathrm{C}, 60.02 ; \mathrm{H}, 3.59 ; \mathrm{N}, 12.56 ; \mathrm{F}$, 3.31; S, 6.74). IR (Nujol mull), $v\left(\mathrm{~cm}^{-1}\right)$ : $3300-2850$ (br,
$\mathrm{OH}$ and $\mathrm{NH}), 1705$ (COOH), 1610 (CONH), 1600, 1570, $1550(3 \mathrm{C}=\mathrm{N}), 1480(\mathrm{Me}), 1360\left(\mathrm{SO}_{2} \mathrm{NH}\right), 1230(\mathrm{C}-\mathrm{F})$. ${ }^{1} \mathrm{H}$ NMR (DMSO- $\mathrm{d}_{6}$ ), $\delta: 14.14$ (s, COOH), 13.0 (s, $\mathrm{CONH}$ ), 11.80 (s, $\mathrm{SO}_{2} \mathrm{NH}$ ), 8.90, 8.70 (each s, $2 \mathrm{H}$, pyrimidine), 8.55 (d, $1 \mathrm{H}, J=7.7 \mathrm{~Hz}$ ), $8.2-8.1$ (m, 2H, $o$-fluorophenyl), 7.85 - 7.78 and 7.55 - 7.41 (each m, 4H, 1,4 disubstituted phenyl), 2.10 (s, $\left.3 \mathrm{H}, \mathrm{CH}_{3}\right) .{ }^{13} \mathrm{C}$ NMR (DMSO- $\left.\mathrm{d}_{6}\right), \delta: 170.1(\mathrm{COOH}), 167.3(\mathrm{NHC}=\mathrm{O}), 151.0$ (C-F), $150.0(\mathrm{C}=\mathrm{N}), 148.1,138.0,136.1,134.3,130.0$, 128.4, 128.1, 127.6, 126.4, 125.3, 124.9, 122.9 (hetero, aryl and phenyl carbons).

6-Fluoro-2-phenyl-3-(arylamino) oxo-quinolines (4a-d). A mixture of compound 3a-d (100 mg) and $\mathrm{K}_{2} \mathrm{CO}_{3}$ solution $(5 \%, 100 \mathrm{ml})$ was heated at $40^{\circ} \mathrm{C}-60^{\circ} \mathrm{C}$ for $10 \mathrm{mins}$, the cooled reaction mixture was neutralized by acetic acids. The yellow solid was collected, washed with cold water, dried and crystallized from dioxan to give 4a-d.

6-Fluoro-2-phenyl-3-[(4'-fluorophenyl)amino]-oxoquinoline (4a). Yellow crystals, Yield $70 \%$; m.p. $260^{\circ} \mathrm{C}$ $262{ }^{\circ} \mathrm{C}$; (Found = C, 72.59; H, 3.83, N, 7.66; F, 10.68, $\mathrm{C}_{22}, \mathrm{H}_{14} \mathrm{~N}_{2} \mathrm{~F}_{2} \mathrm{O}$ requires $\mathrm{C}, 73.13 ; \mathrm{H}, 3.87, \mathrm{~N}, 7.75 ; \mathrm{F}$, 10.80); IR (Nujol mull), $v\left(\mathrm{~cm}^{-1}\right)$ : $3100-3010$ (br, NH and $\mathrm{OH}$ aryl), $1590(\mathrm{CONH}), 1550(\mathrm{C}=\mathrm{N}), 1230(\mathrm{C}-\mathrm{F})$, 880,780 (aryl CH). ${ }^{1} \mathrm{H}$ NMR (DMSO-d 6 ), $\delta: 11.70(\mathrm{~s}, 1 \mathrm{H}$, NHCO) 8.90 (s, $1 \mathrm{H}$, of quinoline-4-yl), 8.5 (d, $1 \mathrm{H}, J=$ $8.1 \mathrm{~Hz}$ ), 8.2 (t, $1 \mathrm{H}, J=7.1 \mathrm{~Hz}) .7 .90$ - 7.83 (m, 4H) 7.53 $7.40(\mathrm{~m}, 3 \mathrm{H})$ and $7.35-6.99(\mathrm{~m}, 5 \mathrm{H}$, aryl and phenyl protons). ${ }^{13} \mathrm{C}$ NMR (DMSO-d 6 ), $\delta: 159.8$ (CONH), 145, 141(C-F), 139.0 (C-N), 136.0, 134.3, 132.5, 130.5, 129.11, 128.3, 127.7, 126.9, 125.1, 124.9, 123.9 (hetero, aryl and phenyl carbons).

6-Fluoro-2-phenyl-3-[(8',4'-dichlorophenyl)amino]oxo-quinoline (4b). Faint Yellow crystals, Yield 68\%; m.p. $195^{\circ} \mathrm{C}-196^{\circ} \mathrm{C}$; (Found = C, 63.75; H, 3.12; N, 6.74; F, 4.56; $\mathrm{Cl}, 17.08, \mathrm{C}_{22} \mathrm{H}_{13} \mathrm{~N}_{2} \mathrm{FCl}_{2} \mathrm{O}$ requires $\mathrm{C}, 64.23 ; \mathrm{H}$, 3.16 N, 6.81; F, $4.62 \mathrm{Cl}, 17.27)$. IR (Nujol mull), $v\left(\mathrm{~cm}^{-1}\right)$ : 
3090 - 3010 (br, NH, C-H arom), 1620 (CONH), 1580 $(\mathrm{C}=\mathrm{N}), 1220$ (C-F), 890, 780 (aromatic CH), 700 (C-Cl) $\mathrm{cm}^{-1} ; \delta_{\mathrm{H}}: 11.2(\mathrm{~s}, 1 \mathrm{H}, \mathrm{NHCO}), 8.9$ (s, $1 \mathrm{H}$ of quinoline-4yl), 8.66 (d, $1 \mathrm{H}, J=8.1 \mathrm{~Hz}), 8.4(\mathrm{t}, 1 \mathrm{H}, J=7.8 \mathrm{~Hz}), 8.2$ (d, $1 \mathrm{H}, J=7.5 \mathrm{~Hz}), 7.90$ - 7.92 (m, 2H), $7.53-7.43$ (m, $5 \mathrm{H}),{ }^{13} \mathrm{C}$ NMR (DMSO-d 6 ), $\delta: 160.1$ (CONH), 142.0 (C-F), 140.8, 140.3 (2 C-Cl), 139.5 (C-N), 133.4, 132.5, $130.1,129.7,128.5,127.3$, 126.9 (hetero, aryl and phenyl carbons).

6-Fluoro-2-phenyl-3-[(4'-heteroaryl-sulfonamidoph enyl)amino]-oxo-quinoline (4c). Yellow crystals, Yield 69\%; m.p. $154^{\circ} \mathrm{C}-156^{\circ} \mathrm{C}$; (Found: C, 59.25; H, 3.33; N, 10.97; F, 3.71; S, 12.52, $\mathrm{C}_{25} \mathrm{H}_{17} \mathrm{~N}_{4} \mathrm{FS}_{2} \mathrm{O}_{3}$ requires: 59.52; H, 3.37; N, 11.11; F, 3.76; S, 12.69); IR (Nujol mull), $v$ $\left(\mathrm{cm}^{-1}\right): 3100$ - 3000 (br, NH, $\mathrm{CH}_{\text {arom}}$ ), 1620 (CONH), $1550(\mathrm{C}=\mathrm{N}), 1520,1350$ (NCS and $\left.\mathrm{SO}_{2} \mathrm{NH}\right), 1250(\mathrm{C}-\mathrm{F})$, 870, $770\left(\mathrm{CH}_{\text {arom }}\right), 710$ (C-F). ${ }^{1} \mathrm{H}$ NMR (DMSO-d 6 ), $\delta$ : 11.81 (s, 1H, CONH), 8.91, 8.73 (d, 2H, thiazole), 8.40 8.34 (d, $1 \mathrm{H}, J=8.3 \mathrm{~Hz}$ ), 8.20 - 8.10 (m, 2H, $o$-fluorophenyl), 7.81 - 7.71 and 7.55 - 7.35 (each $\mathrm{m}, 4 \mathrm{H}$, 1,4-disubstituted phenyl). ${ }^{13} \mathrm{C}$ NMR (DMSO-d 6 ), $\delta: 162.1$ (CONH), 151.9 (N-C-S), 150.9, 150.1(C-F and C-N), 147.9, 138.3, 137.0. 136.5, 135.0, 134.1, 132.3, 129.8, 128.7, 127.4, 126.1, 125.8 (hetero, aryl and phenyl carbons).

6-Fluoro-2-phenyl-3-[(4'-heteroaryl-sulfonamidoph enyl)amino]-oxo-quinoline (4d). Orange crystals, Yield $75 \%$; m.p. $158^{\circ} \mathrm{C}-160^{\circ} \mathrm{C}$; (Found: C, 62.66; H, 3.84; $\mathrm{N}, 13.48 ; \mathrm{F}, 3.61 ; \mathrm{S}, 6.16, \mathrm{C}_{27}, \mathrm{H}_{20} \mathrm{~N}_{5} \mathrm{FSO}_{3}$ requires: $\mathrm{C}$, 63.15; H, 3.89; N, 13.64; F. 3.70; S. 6.23). IR (Nujol mull), $v\left(\mathrm{~cm}^{-1}\right): 3090$ - 3000 (br, $\left.\mathrm{NH}, \mathrm{CH}_{\text {arom }}\right), 1590$ $(\mathrm{CONH}), 1580(\mathrm{C}=\mathrm{N}), 1448$ (deformation $\left.\mathrm{CH}_{3}\right), 1330$ $\left(\mathrm{SO}_{2} \mathrm{NH}\right), 1250$ (C-F), 850, 780 (aromatic $\mathrm{CH}$ ) and 700 (C-F). ${ }^{1} \mathrm{H}$ NMR (DMSO-d $\mathrm{d}_{6}$ ), $\delta: 12.10$ (s, 1H, CONH), 8.91 (s, $1 \mathrm{H}$ of quinolin-4-yl), 8.53 (d, $1 \mathrm{H}, J=8.2 \mathrm{~Hz}$ ), 8.20 (t, 1H, $J=7.11 \mathrm{~Hz}$ ), 7.89 - 7.85 (m, 2H), 7.46 - 7.43 (m, 3H, aryl and aromatic protons). ${ }^{13} \mathrm{C}$ NMR (DMSO$\mathrm{d}_{6}$ ), $\delta$ : 162.5 (CONH), 150.2, 150.6, 150.00 (C-F, C-N, $\mathrm{N}-\mathrm{C}-\mathrm{S}$ ), 148.5 (C-4, quinoline) 138.1, 136.3, 135.4, 134.0, 132.8, 130.7, 128.8, 128.1, 127.0, 126.3, 125.3, 124.9, 123.0 (hetero aryl and phenyl carbons).

7-Fluoro-1-aryl-3-phenyl-pyrrolo-[3,4-c]-quinoline2,9-diones (5a-d). A mixture of compounds 3a-d (100 $\mathrm{mg})$ and $\mathrm{K}_{2} \mathrm{CO}_{3}$ solution $(5 \%, 100 \mathrm{ml})$ was first stirred at room temperature for $1 \mathrm{~h}$, the reaction was continue at $80^{\circ} \mathrm{C}-100^{\circ} \mathrm{C}$ for further $2 \mathrm{~h}$, after cooling, the reaction mixture was neutralized by acetic acid $(30 \% \mathrm{v} / \mathrm{v})$, The produced solid was filtered, washed with cold water, dried and crystallized from dioxan to give 5a-d.

7-Fluoro-1-(4'-fluorophenyl)-3-phenyl-pyrrolo [3,4-c] quinoline-2,9-dione (5a). Faint yellow crystals, Yield $65 \%$; m.p. $230^{\circ} \mathrm{C}-232^{\circ} \mathrm{C}$; (Found = C, 70.89; H, 3.06; N, 7.05; F, 9.89, $\mathrm{C}_{23} \mathrm{H}_{12} \mathrm{~N}_{2} \mathrm{~F}_{2} \mathrm{O}_{2}$ requires C, 71.31; H, 3.10;
N, 7.23, F, 10.07; IR (Nujol mull), $v\left(\mathrm{~cm}^{-1}\right)$ : 3000 (aromatic $\mathrm{CH}), 1710,1680(2 \mathrm{C}=\mathrm{O}), 1580(\mathrm{C}=\mathrm{N}) 1350$ ( $\left.\mathrm{SO}_{2} \mathrm{NH}\right) 1250(\mathrm{C}-\mathrm{F}), 870,820$ (p-substituted phenyl). ${ }^{1} \mathrm{H}$ NMR (DMSO-d d $_{6}, \delta: 8.21$ (d, $\left.1 \mathrm{H}, J=7.5 \mathrm{~Hz}\right) 7.90-7.88$ (m, 2H, hetero protons), $7.70-7.38$ and $7.14-6.94$ (each, $\mathrm{m}, 4 \mathrm{H}$ and $\mathrm{SH}$ aryl and phenyl protons). ${ }^{13} \mathrm{C} \mathrm{NMR}$ (DMSO-d $\mathrm{d}_{6}$ ), $\delta$ : 170.1, 168.1 (2 C=O), 151.6 and 151.1 (C-F), 141.0 (C-N), 136.5, 135.1, 134.8, 132.7, 130.8, 128. 7, 127.9, 126.3, 125.1, 124.0, 122.0 (hetero, aryl and phenyl carbons).

7-Fluoro-1-(2',4'-dichlorophenyl)-3-phenyl pyrrole [3,4-c] quinoline-2,9-dione (5b). Yellow crystals, Yield 68\%; m.p. $218^{\circ} \mathrm{C}-220^{\circ} \mathrm{C}$; (Found: C, 62.75; H, 2.47; N, 6.30; F, 4.29; $\mathrm{Cl}$ 16.07, $\mathrm{C}_{23} \mathrm{H}_{11} \mathrm{~N}_{2} \mathrm{FCl}_{2} \mathrm{O}_{2}$ requires: $\mathrm{C}$, 63.15; H, 2.15; N, 6.40; F, 4.34; Cl, 16.24). IR (Nujol mull), $v\left(\mathrm{~cm}^{-1}\right)$ : 3010 ( $\left.\mathrm{CH}_{\text {arom }}\right), 1700,1680$ (2 $\left.\mathrm{C}=\mathrm{O}\right)$, $1580(\mathrm{C}=\mathrm{N}), 1250$ (C-F), 850, 820 (substituted phenyl), 710 (C-Cl). ${ }^{1} \mathrm{H}$ NMR (DMSO-d $\left.{ }_{6}\right), \delta: 8.11$ (d, $1 \mathrm{H}, J=7.8$ $\mathrm{Hz}), 7.83$ - 7.74 (m, 2H), 7.64 - 7.52 (m, 2H), 7.21 - 7.18 (aromatic protons). ${ }^{13} \mathrm{C}$ NMR (DMSO- $\left.\mathrm{d}_{6}\right), \delta: 172.2$, 169.5 (2 C=O), 150.3 (C-F), 146, 146.1 (2 C-Cl), 140.4 (C-N), 135.8, 134.1, 132.8, 130.1, 128.9, 127.6, 126.1, $125.9,124.8,123.0$ (hetero aryl and phenyl protons).

7-Fluoro-1-(4'-heteroaryl sulfanamidophenyl)-3phenyl pyrrolo (3,4-c) quinoline-2,9-dione (5c). Deep yellow crystals, $66 \%$. M.p. $165^{\circ} \mathrm{C}-166^{\circ} \mathrm{C}$. (Found: C, 60.39; H, 2.85; N, 10.77; F, 3.64; S, 12.31, $\mathrm{C}_{26}$, $\mathrm{H}_{15} \mathrm{~N}_{4} \mathrm{FS}_{2} \mathrm{O}_{3}$ requires: $\mathrm{C}, 60.70 ; \mathrm{H}, 2.91 ; \mathrm{N}, 10.89 ; \mathrm{F}$, 3.69, S, 12.45 ); IR (Nujol mull), $v\left(\mathrm{~cm}^{-1}\right)$ : $3000-2990$ $\left(\mathrm{CH}_{\text {arom }}\right), 1690,1680(2 \mathrm{C}=\mathrm{O}), 1605(\mathrm{C}=\mathrm{N}), 1380$ ( $\left.\mathrm{SO}_{2} \mathrm{NH}\right), 1230$ (C-F), 870, 790 (substituted phenyl). ${ }^{1} \mathrm{H}$ NMR (DMSO- $\left.\mathrm{d}_{6}\right), \delta$ : $8.9\left(\mathrm{NHSO}_{2}\right), 8.55$ (d, 2H, thianzole), 8.40, 8.28 (d, $1 \mathrm{H}, J=7.9 \mathrm{~Hz}$ ), $8.20-8.12$ (m, $2 \mathrm{H}$ of $o$-fluorophenyl), $7.70-7.51(\mathrm{~m}, 9 \mathrm{H}$, aryl and phenyl protons). ${ }^{13} \mathrm{C}$ NMR (DMSO- $\left.\mathrm{d}_{6}\right), \delta: 174,172(2 \mathrm{C}=\mathrm{O})$, 158.0 (N-C-S), 150.9, (C-F), $150.1(\mathrm{C}=\mathrm{N}), 148.1(\mathrm{C}-\mathrm{N})$, 138.1, 137.5, 136.5, 134.9, 132.0, 128.7, 127.9, 126.6, 125.4, 124.1, 122.2 (hetero, aryl and phenyl carbons).

7-Fluoro-1-(4'-heteroaryl sulfanamidophenyl)-3-phenyl pyrrolo [3,4-c] quinoline-2,9-dione (5d). Yellow crystals, Yield 65\%, m.p. $132^{\circ} \mathrm{C}-135^{\circ} \mathrm{C}$; (Found: C, 63.69; $\mathrm{H}, 3.37$; N, 13.23; F. 3.59; S, 6.04, $\mathrm{C}_{28}, \mathrm{H}_{18} \mathrm{~N}_{5} \mathrm{FSO}_{3}$ requires C, 64.24; H, 3.44; N, 13.38; F, 3.63; S, 6.11), IR (Nujol mull), $v\left(\mathrm{~cm}^{-1}\right)$ : 3010, $2985\left(\mathrm{CH}_{\text {arom }}\right), 1705,1690$ (2 $\mathrm{C}=\mathrm{O}), 1590(\mathrm{C}=\mathrm{N}), 1480$ (deformation $\mathrm{CH}_{3}$ ), 1340 ( $\left.\mathrm{SO}_{2} \mathrm{NH}\right) .1250$ (C-F), 850, 820 (substituted phenyl). ${ }^{1} \mathrm{H}$ NMR (DMSO- $\mathrm{d}_{6}$ ), $\delta: 8.80\left(\mathrm{~s}, 1 \mathrm{H}, \mathrm{SO}_{2} \mathrm{NH}\right), 8.33$ and 8.21 (each t, $1 \mathrm{H}, J=7.9$ and $7.6 \mathrm{~Hz}) 7.91-7.85(\mathrm{~d}, 2 \mathrm{H}$, pyrimidine) 7.41 - 7.38 (m, 4H), 7.22 - 7.10 (m, 5H, Aryl and phenyl protons). ${ }^{13} \mathrm{C}$ NMR (DMSO- $\left.\mathrm{d}_{6}\right), \delta: 171,168.9$ $(2 \mathrm{C}=)), 151.1(\mathrm{C}-\mathrm{F}), 150.3(\mathrm{C}=\mathrm{N}), 149.1(\mathrm{C}=\mathrm{N}), 138$, 137.7 (2 C-N), 132.6, 130.1, 128.7, 128.1, 126.9, 125.7, 124.5 and 123.0 (hetero aryl and phenyl carbons). 


\section{Acknowledgements}

We wish to express our thanks to Dr. Ismaa N. Ismail, Department of Biochemistry, Faculty of Science, Ain Shams University, Cairo, for her help in amylolytic evaluation in this study.

\section{REFERENCES}

[1] D. E. Davidson, A. L. Agas, J. L. Brown, F. E. Chapple, R. E. Whitmire and R. N. Rossan, "New Tissue Schizontocidal Antimalarial Drugs," Bulletin of the World Health Organization, Vol. 59, No. 3, 1981, pp. 463-471.

[2] M. Kidwai, N. Gupta and K. C. Srivastava, "Synthesis and Antiviral Activity of 3(4'-Methyl Quinolino-2'-Yloxo) 2H Benzopyran-2-Ones,” Indian Drugs, Vol. 30, 1993, pp. 377-380.

[3] M. Kidwai, N. Negi and P. Misra, "Microwave Assisted Synthesis of 2,3-Disubstituted-4-thiazolidinones and Their Pharmacological Screening," Journal of the Indian Chemical Society, Vol. 77, 2000, pp. 46-47.

[4] M. Baba, M. Okamoto, M. Makino, Y. Kimura, T. Ikenchi, T. Sakaguchi and T. Okamoto, "Potent and Selective Inhibition of Human Immunodeficiency Virus Type 1 Transcription by Piperazinyloxoquinoline Derivatives," Antimicrobial Agents and Chemotherapy, Vol. 41, No. 6, 1997, pp. 1250-1255.

[5] M. Baba, M. Okamoto, M. Kawamura, M. Makino, T. Highashida, T. Takashi, Y. Kimura, T. Ikeuchi, T. Tetsuka and T. Okamoto, "Inhibition of Human Immunodeficiency Virus Type 1 Replication and Cytokine Production by Fluoroquinoline Derivatives," Molecular Pharmacology, Vol. 53, No. 6, 1998, pp. 1097-1103.

[6] H. Okamoto, T. P. Cujec, M. Okamoto, B. M. Peterlin, M. Baba and T. Okamoto, "Inhibition of the RNA-Dependent Transactivation and Replication of HIV 1 by A Fluoroquinoline Derivative K-37," Virology, Vol. 272, No. 2, 2000, pp. 402-408. doi:10.1006/viro.2000.0396

[7] E. De Clereq, "New Developments in Anti-HIV Chemotherapy," Biochimica et Biophysica Acta, Vol. 1587, No. 2-3, 2002, pp. 258-275.

[8] X. Wang, K. Yamataka, M. Okamoto, S. Ikeda and M. Baba, "Potent and Selective Inhibition of Tat-Dependent HIV-1 Replication in Chronically Infected Cells by a Novel Naphthalene Derivative JTK-101,” Antimicrobial Agents and Chemotherapy, Vol. 18, 2007, pp. 201-211. doi:10.1021/ol006936u

[9] H. Amii, Y. Kishikaws and K. Umeyama, "Rh(I)-Catalyzed Cyclization Coupling of N-Aryl Trifluoroacetimidoyl Chlorides with Alkynes: One-pot Synthesis of Fluorinated Quinolines,” Organic Letters, Vol. 3, No. 8, 2001, pp. 1109-1112.

[10] C. S. Cho, B. H. Oh, J. S. Kim, T. S. Kim and S. C. Shim, "Synthesis of Quinolines via Ruthenium-Catalysed Amine Exchange Reaction between Anilines and Trialkylamines," Chemical Communications, No. 19, 2000, pp. 18851886. doi:10.1039/b005966h

[11] N. D. Obushak, N. T. Pokhodylo, I. T. Krupa and V. S.
Matiichuk, "Synthesis of Substituted 4-([1,2,4]Triazolo[3,4-b]-[1,3,4]thiadiazol-6-yl)quinolines,” Russian Journal of Organic Chemistry, Vol. 43, No. 8, 2007, pp. 1223-1227. doi:10.1134/S1070428007080246

[12] M. G. Shevekhgeimer and N. N. Kondrashora, "Synthesis of New Derivatives of 4-Quinolinecarboxylic Acid," Heterocyclic Chemistry, Vol. 38, No. 2, 2002, pp. 253254. doi:10.1023/A:1015363931038

[13] X. Y. Yu, J. M. Hil, G. Yang, Y. Yu, A. F. Kluge, D. Keith, J. Finn, P. Gallant, J. Silverman and A. Lim, “A Series of Quinoline Analogues as Potent Inhibitors of $C$. albicans Prolyl tRNA Synthetase," Bioorganic \& $\mathrm{Me}$ dicinal Chemistry Letters, Vol. 11, No. 4, 2001, pp. 541-544. doi:10.1016/S0960-894X(00)00697-1

[14] Y. Kurasawa, K. Yoshida, N. Yamazaki, E. Kaji, K. Sasaki, Y. Zamami, Y. Sakai, T. Fujii and H. Ito, "Quinolone Analogs 11: Synthesis of Novel 4-Quinolone3-carbohydrazide Derivatives with Antimalarial Activity,” Journal of Heterocyclic Chemistry, 2011.

[15] A. Wube, F. Bucar, C. Hochfellner, M. Blunder, R. Bauer and A. Hüfner, "Synthesis of N-Substituted 2-[(1E)-Alkenyl]-4-(1H)-Quinolone Derivatives as Antimycobacterial Agents against Non-Tubercular Mycobacteria," European Journal of Medicinal Chemistry, Vol. 46, No. 6, 2011, pp. 2091-2101. doi:10.1016/j.ejmech.2011.02.062

[16] M. A. Ibrahim, H. M. Hassanin, Y. A. Gabr and Y. AlNamer, "Novel Heterocyclic Derivatives of Pyrano[3,2c]quinolinone from 3-(1-Ethy1-4-hydroxy-2-oxo-2(1H)quinolin-3-yl)-3-oxopropanoic Acid,” European Journal of Chemistry, Vol. 1, No. 3, 2010, pp. 195-199. doi:10.5155/eurjchem.1.3.195-199.91

[17] E. A. Mohammed, R. M. Abdle-Rahman, A. A. Seayed and M. Ismail, "Synthesis of 3-Heteroaryl-4-hydroxybenzocarbostyrils," Journal of the Indian Chemical Society, Vol. 69, No. 2, 1992, pp. 472-475.

[18] E. A. Mohammed, R. M. Abdel-Rahman, A. M. Tawfik and M. Ismail, "Behavior of Some 3-Formyl-4-hydroxy Carboxylic towards Amines, Hydrazines and Hydroxylamine," Pakistan Journal of Science and Industrial Research, Vol. 36, 1993, pp. 223-227.

[19] E. A. Mohammed, R. M. Abdel-Rahman, Z. El-Gendy and M. Ismail, "Some Reactions of 3-Formyl-1,8-naphthyl Ridine-2-one,” Annales de Quimica, Vol. 89, No. 2, 1993, pp. 246-253.

[20] E. Kawsari and M. M. Mohammadi, "Ultrasound Promoted Synthesis of Quinolines Using Basic Ionic Liquids in Aqueous Media as a Green Procedure," Ultrasonics Sonochemistry, Vol. 18, No. 1, 2011, pp. 447-454. doi:10.1016/j.ultsonch.2010.07.020

[21] S. Abu-El-Wafa, R. M. Abdel-Rahman and Z. El-Gendy, "Spectroscopic Studies of Some Antilide Compounds Derived from Ethyl Benzoyl Acetate,” Egyptian Journal of Chemistry, Vol. 33, No. 5, 1990, pp. 387-395.

[22] R. M. Abdel-Rahman, "Synthesis and Anti Human Immune Virus Activity of Some New Fluorine Containing Substituted-3-thioxo-1,2,4-triazin-5-ones,” Farmaco, Vol. 46, 1991, pp. 379-389.

[23] R. M. Abdlel-Rahman, "Synthesis of Some New Fluorine 
Bearing Trisubstituted 3-Thioxo-1,2,4-triazin-5-ones as Potential Anticancer Agents,” Farmaco, Vol. 47, No. 3, 1992, pp. 319-326;

[24] R. M. Abdel-Rahman, "Role of Uncondensed 1,2,4-Triazine Compounds and Related Heterocyclic Systems as therapeutic agents. A Review, Part XV,” Pharmazie, Vol. 56, No. 1, 2001, pp. 18-22;

[25] R. M. Abdel-Rahman, "Role of Uncondensed 1,2,4-Triazine Derivatives as Biocidal Plant Protection Agents," Pharmazie, Vol. 56, No. 1, 2001, pp. 195-204.

[26] R. M. Abdel-Rahman and M. S. Abdel-Malik, "Synthesis of Some New 3,6-Diheteroaryl-1,2,4-triazin-5-ones and Their Effect on Amylolytic Activity of Some Fungi," Pakistan Journal of Science and Industrial Research, Vol. 33, No. 4, 1990, pp. 142-147.

[27] N. Nelson, "A Photometric Adaptation of the Somogyi Method for the Determination of Glucose,” Journal of Biological Chemistry, Vol. 153, No. 2, 1944, pp. 375380.

[28] M. Somogyi, "A New Reagent for the Determination of Sugars,” Journal of Biological Chemistry, Vol. 160, 1945, pp. 61-68.

[29] S. Brautigam, D. Dennewald, M. Schurmann, J. LutjeSpelberg, W. Pitner and D. Weuster-Botz, "Whole-Cell Biocatalysis: Evaluation of New Hydrophobic Ionic Liquids for Efficient Asymmetric Reduction of Prochiral Ketones," Enzyme and Microbial Technology, Vol. 45, No. 4, 2009, pp. 310-316. doi:10.1016/j.enzmictec.2009.06.015

[30] R. Gupta, P. Gigras, H. Mohapatra, V. K. Goswami and B. Chauhan, "Microbial $\alpha$-Amylses: A Biotechnological Prespective,” Process Biochemistry, Vol. 38, No. 11, 2003, pp. 1-18.

[31] B. K. Park, N. R. Kitteringham and P. M. O’Neill, "Metabolism of Fluorine-Containing Drugs," Annual Review of Pharmacology and Toxicology, Vol. 41, 2001, pp. 443-470. doi:10.1146/annurev.pharmtox.41.1.443

[32] R. Filler and R. Saha, "Fluorine in Medicinal Chemistry: A Century of Progress and a 60-Year Retrospective of Selected Highlights," Future Medicinal Chemistry, Vol. 1, No. 5, 2009, pp. 777-791. doi:10.4155/fmc.09.65 